\title{
Readiness to Change in Primary Care Patients Who Screened Positive for Alcohol Misuse
}

\author{
Emily C. Williams, $\mathrm{MPH}^{1-3}$ \\ Daniel R. Kivlaban, $P b D^{2,4}$ \\ Richard Saitz, MD, $M P H^{5,6}$ \\ Joseph O. Merrill, MD, MPH ${ }^{3,7}$ \\ Carol E. Acbtmeyer, $M^{1,2,8}$ \\ Kinsey A. McCormick, BA ${ }^{1,3}$ \\ Katharine A. Bradley, MD, MPH ${ }^{1-3,8,9}$ \\ 'Health Services Research \& Development, \\ VA Puget Sound Health Care System, \\ Seattle, Wash \\ ${ }^{2}$ Center of Excellence in Substance Abuse
} Treatment and Education, VA Puget Sound Health Care System, Seattle, Wash

${ }^{3}$ Department of Medicine, School of Medicine, University of Washington, Seattle, Wash

${ }^{4}$ Departments of Psychiatry and Behavioral Sciences, University of Washington, Seattle, Wash

${ }^{5}$ Clinical Addiction Research and Education Unit, Department of Medicine, Boston University School of Medicine, and Boston Medical Center, Boston, Mass

${ }^{6}$ Youth Alcohol Prevention Center and Department of Epidemiology, Boston University School of Public Health, Boston, Mass

${ }^{7}$ Harborview Medical Center, Seattle, Wash

${ }^{8}$ Primary and Specialty Medical Care Service, VA Puget Sound Health Care System, Seattle, Wash

${ }^{9}$ Department of Health Services, University of Washington, Seattle, Wash

Conflicts of interest: none reported

\section{CORRESPONDING AUTHOR}

Emily C. Williams, MPH

VA Puget Sound Health Care System 1100 Olive Way, Suite 1400

Seattle, WA 98101

emwilli@u.washington.edu

\begin{abstract}
PURPOSE Readiness to change drinking may influence the content or effectiveness of brief alcohol counseling. This study was designed to assess readiness to change and its relationship to alcohol misuse severity among primary care patients whose screening questionnaire was positive for alcohol misuse.
\end{abstract}

METHODS This study was a cross-sectional analysis of data collected from 2 consecutive mailed questionnaires. Male outpatients at 7 Veterans Affairs (VA) general medicine clinics were eligible if they returned both questionnaires, screened positive for alcohol misuse (augmented CAGE Questionnaire $\geq 1$ point), responded to 3 readiness-to-change questions, and completed the Alcohol Use Disorders Identification Test (AUDIT). A validated algorithm based on 3 standardized questions categorized participants into 3 readiness groups (precontemplation, contemplation, action). Measures of alcohol misuse severity included AUDIT, CAGE, and the 3 consumption questions from the AUDIT (AUDIT-C). Analyses were descriptive; linear-by-linear associations between alcohol misuse severity and readiness were tested with $\chi^{2}$ statistics.

RESULTS Response rates to the first and second surveys were $59 \%$ and $55 \%$, respectively. Of the 6,419 eligible outpatients who screened positive for alcohol misuse, $4,797(75 \%)$ reported any readiness to change (contemplation $24 \%$, action 51\%). Among patients with AUDIT scores $>8$, more than $90 \%$ indicated that they drank more than they should and/or had contemplated drinking less. Greater readiness was significantly associated with greater alcohol misuse severity $(P<.001$ for all measures).

CONCLUSIONS Most primary care patients who screen positive for alcohol misuse indicate some readiness to change. Contrary to stereotypes of denial, those with greater alcohol misuse severity are more likely to report readiness to change.

Ann Fam Med 2006;4:213-220. DOI: 10.1370/afm.542.

\section{INTRODUCTION}

$\Lambda$ lcohol misuse is common and among the most costly public health problems in the United States. ${ }^{1,2}$ Brief counseling interventions reduce drinking in patients who screen positive for alcohol misuse. ${ }^{3}$ The US Preventive Services Task Force recommends routine alcohol screening in primary care settings and brief alcohol counseling for patients who screen positive for alcohol misuse. ${ }^{4}$ Though the exact content of brief alcohol counseling interventions evaluated in randomized trials has varied, these interventions typically include patient-centered assessment, individualized feedback and advice, agreement on a drinking goal, and follow-up. ${ }^{5}$ These interventions last approximately 5 to 15 minutes when delivered in primary care settings. ${ }^{6}$

As with counseling about other health behaviors, such as tobacco use, when clinicians counsel patients about drinking, the appropriate focus of the discussion and patients' receptivity can depend on patients' readiness to change. ${ }^{7.9}$ For example, the content of brief counseling should reflect whether the patient recognizes his drinking is a problem and whether he 
has contemplated or tried to change. ${ }^{10-12}$ One study suggested that the efficacy of brief alcohol counseling differed for patients with differing readiness to change. ${ }^{11}$ Further, clinicians have reported concern that primary care patients who screen positive for alcohol misuse will deny that they misuse alcohol or will not be interested in discussing or changing their drinking when the issue is raised..$^{13-16}$ Denial of alcohol misuse is sometimes assumed to be a characteristic of alcohol dependence. ${ }^{14}$

Despite its clinical utility, little research has addressed the prevalence of denial or readiness to change among primary care patients who misuse alcohol. ${ }^{11,17,18}$ No study has described readiness to change in a large sample of primary care patients who screened positive for alcohol misuse but were not recruited into a study focused exclusively on alcohol misuse. This study describes readiness to change and evaluates its relationship to alcohol misuse severity in more than 6,000 outpatients who screened positive for alcohol misuse as part of a quality improvement study addressing multiple common outpatient conditions.

\section{METHODS}

\section{Setting and Population}

General internal medicine outpatients from 7 Veterans Affairs (VA) sites (Birmingham, Ala, Little Rock, Ark; San Francisco, Calif; West Los Angeles, Calif; White River Junction, $\mathrm{Vt}_{\text {; }}$ Richmond, $\mathrm{Va}_{\text {; }}$ and Seattle, Wash) were surveyed by mail as part of the VA Ambulatory Care Quality Improvement Project (ACQUIP). ${ }^{19}$ The ACQUIP trial was a quality improvement study evaluating the effectiveness of giving clinicians individualized feedback based on patient questionnaires regarding 6 medical conditions (coronary artery disease, diabetes, chronic obstructive pulmonary disease, hypertension, depression, and alcohol misuse). A representative sample of 62,487 patients who visited a participating general internal medicine clinic from 1997 to 2000 received a mailed Health Checklist, which included a validated 8 -item screen consisting of the CAGE Questionnaire (cut down, annoyed, guilty, and eye opener), combined with 4 additional questions about alcohol consequences and previous problem drinking. ${ }^{20}$ Potential ACQUIP participants were identified through medical records review. A waiver of written informed consent was obtained along with study approval from the University of Washington Human Subjects Committee and the institutional review boards at each site.

Patients who returned this Health Checklist and who had a screening questionnaire that was positive for alcohol misuse $(\geq 1 \text { point })^{20}$ on the augmented
CAGE Questionnaire were mailed a subsequent survey instrument, the Drinking Practices Questionnaire, to further assess alcohol use. This instrument included the 10-item Alcohol Use Disorders Identification Test (AUDIT) and 3 readiness-to-change questions (both measures described below). Patients were also mailed up to 5 other condition-specific questionnaires if they had indicated they had these conditions. A generic cover letter was enclosed with the condition-specific questionnaires indicating that patient responses might be shared with their primary care clinicians as part of a study designed to determine whether such information improved care. There was no alcohol-specific information in the cover letter.

Male respondents to the Drinking Practices Questionnaire who reported drinking in the past year and completed the readiness-to-change, AUDIT, and CAGE questions were included in this study. The few female respondents were excluded because of sex differences in the performance of alcohol-screening questionnaires ${ }^{21}$ and insufficient variation in alcohol misuse severity among participating women to conduct sex-specific analyses.

\section{Measures}

\section{Readiness to Change}

Readiness to change has often been categorized into 3 stages (precontemplation, contemplation, and action)

based on Prochaska and DiClemente's transtheoretical model. ${ }^{11,18,22-27}$ Although contemplation was later broken down into component stages, the original transtheoretical model divided patients into 3 groups: those who had no recognition that they drank more than they should and who were not trying to change, those who had some recognition of drinking excessively and who were often contemplating change, and those taking steps to change. ${ }^{23}$ In our study, readiness to change was measured using a brief algorithm based on 3 standardized questions (Figure 1). ${ }^{28}$ This algorithm categorizes patients who misuse alcohol into precontemplation, contemplation, or action groups based on the transtheoretical model. ${ }^{29}$ The questions are designed to guide clinician interventions with patients who screen positive for alcohol misuse and to provide information about changes in drinking at follow-up visits. The questions address any recent changes in the patient's drinking (past 3 months), patient self-recognition of excessive drinking, and whether the patient had considered changing his drinking. In addition to face validity, the readiness-to-change algorithm had good concurrent validity when compared with the longer Readiness to Change Questionnaire by Rollnick et $\mathrm{al}^{3}{ }^{30}$ which was validated in a population of female VA patients who screened positive for alcohol misuse. ${ }^{28}$ 
Measures of Alcohol Misuse Severity

Our primary measure of alcohol misuse severity was the AUDIT, a validated alcohol-screening questionnaire specifically developed to identify patients across the spectrum of alcohol misuse ${ }^{31}$ and validated in a male VA outpatient population. ${ }^{32}$ The AUDIT scores range from 1 to 40 for drinkers, with higher scores indicating greater severity. ${ }^{33}$ The World Health Organization recommends the use of 4 severity zones on the AUDIT $(<8,8-15,16-19, \geq 20)$. Brief alcohol counseling is recommended for those with AUDIT scores $\geq 8$ but $<20$. $^{33}$

Patients with high AUDIT scores are those willing to report past-year problems caused by drinking, which may, in itself, reflect increased recognition or willingness to consider changing drinking. We therefore evaluated the association between readiness to change and 2 scores of other brief alcohol-screening questionnaires that do not ask explicitly about problems caused by drinking in the past year: the 3 AUDIT consumption questions (AUDIT-C) and the CAGE Questionnaire.

The first 3 questions of the 10 -item AUDIT, the AUDIT-C, address alcohol consumption patterns in the past year and have been validated as a brief alcohol-screening test. AUDIT-C scores range from 1 to 12 for drinkers, and a score of 4 or more is effective for screening for alcohol misuse in men. ${ }^{34}$ Typical con-

Figure 1. Readiness to change of patients enrolled in the study.

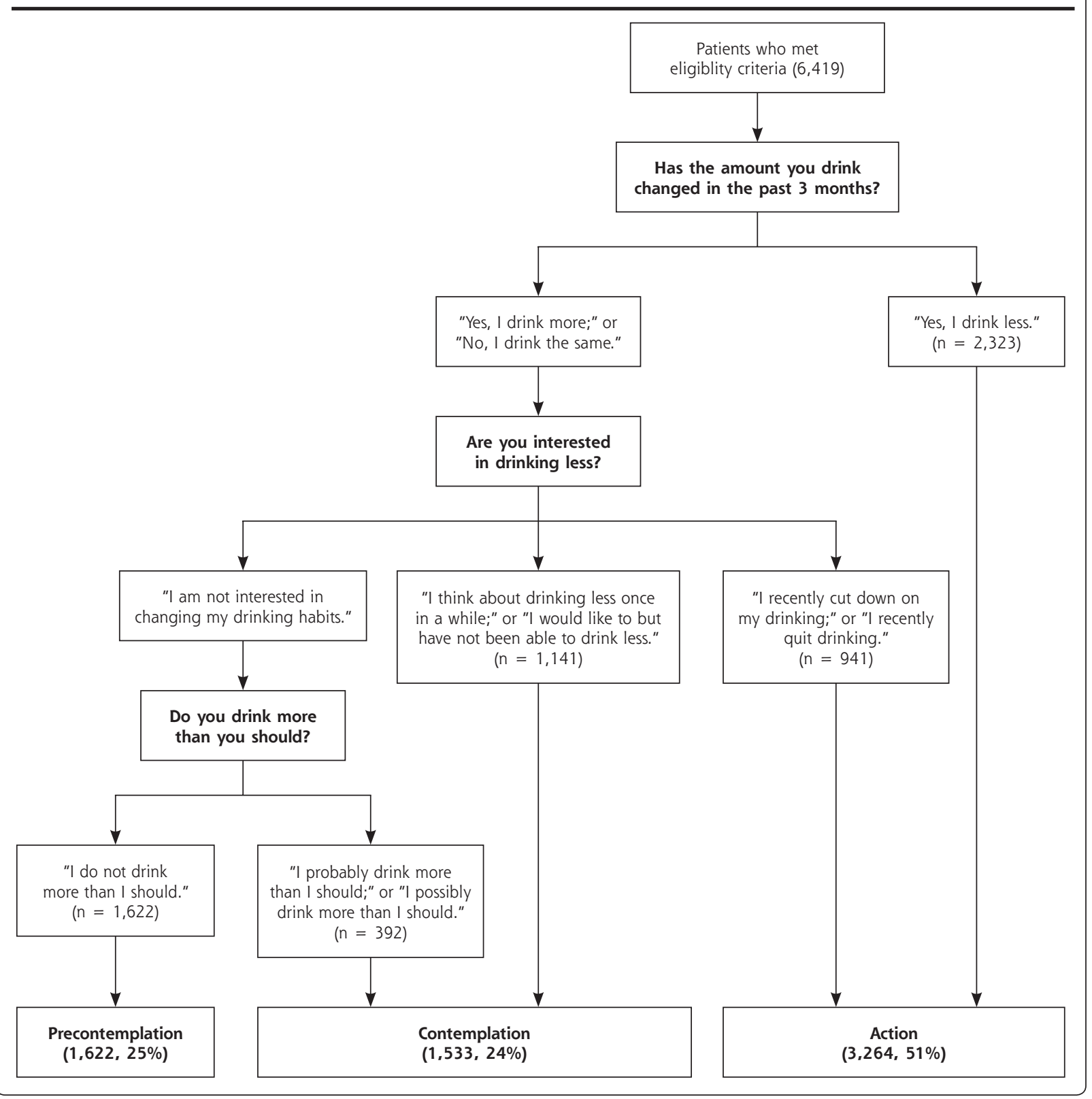


sumption reported on these questions is imprecise and on average underestimates actual consumption, ${ }^{35}$ but increasing scores are related to increasing severity of self-reported alcohol-related problems in the past year. ${ }^{36}$

The 4-item CAGE is an effective screening test for active alcohol abuse and/or dependence (possible scores 0 to 4 ) but is less sensitive for detecting patients with milder alcohol misuse. ${ }^{32,37} \mathrm{~A}$ "yes" response to any CAGE question (score $\geq 1$ ) indicates a positive finding for active alcohol abuse and/or dependence in this population, ${ }^{32}$ although $\geq 2$ has often been used. ${ }^{38}$ The CAGE score is associated with increasing severity of problems caused by drinking in the past year reported on the AUDIT, but the relationship is weaker than that with AUDIT-C scores. ${ }^{36}$

\section{Demographic Characteristics}

Patient age and race were obtained from VA electronic medical records. Other demographic characteristics including income, education, and marital status were obtained from the ACQUIP questionnaires.

\section{Analyses}

Descriptive analyses assessed demographic and clinical characteristics, alcohol-screening results, and the prevalence of precontemplation, contemplation, and action in the study sample of ACQUIP participants who screened positive for alcohol misuse on the augmented CAGE Questionnaire and completed the readiness-tochange questions. To evaluate nonresponse bias, demographic characteristics and alcohol misuse severity measures from the Health Checklist were cross-tabulated for study participants and nonparticipants, and Pearson $\chi^{2}$ statistics were obtained to identify differences.

Analyses first evaluated the association of readiness to change with alcohol misuse severity as measured by the AUDIT. The proportion of patients with each AUDIT score who were in the contemplation or action group was evaluated and depicted graphically. Linear-by-linear $\chi^{2}$ tests were then completed to test the association between readiness groups and all alcohol misuse severity measures $(\alpha=.05)$. To explore further the association of readiness to change with the severity of alcohol misuse based on the AUDIT, we evaluated responses to each of the 3 readiness-to-change questions within individual readiness groups (precontemplation, contemplation, and action). All analyses were carried out using SPSS Version 12.0. ${ }^{39}$

\section{RESULTS}

More than one half the patients returned the Health Checklist $(32,821 ; 59 \%$ of those eligible) and 11,889 (36\% of respondents) screened positive for alcohol misuse $(\geq 1 \text { point })^{20}$ on the augmented CAGE; of these, 6,551 patients $(55 \%$ of those eligible for this survey, $10 \%$ of the initial study population) completed the Drinking Practices Questionnaire. There were 6,419 male respondents to the Drinking Practices Questionnaire who reported drinking in the past year and who completed the readiness-to-change, AUDIT, and CAGE questions.

The characteristics of the 6,419 participants are displayed in Table 1. These respondents represented 55\% of the men who screened positive for alcohol misuse on the initial Health Checklist. When these participants were compared with male patients who screened positive for alcohol misuse was positive but did not respond to the Drinking Practices Questionnaire $(\mathrm{n}=4,815)$ or

\begin{tabular}{|c|c|}
\hline Characteristic & Value \\
\hline Age in years, mean (SD) & $60(11.5)$ \\
\hline \multicolumn{2}{|l|}{ Race, ${ }^{*}$ No. $(\%)$} \\
\hline African American & 1,199 (19) \\
\hline White & $4,081(64)$ \\
\hline Other & $981(15)$ \\
\hline \multicolumn{2}{|l|}{ Marital status, ${ }^{\dagger}$ No. (\%) } \\
\hline Never married & 597 (9) \\
\hline Currently married & $3,163(49)$ \\
\hline Divorced/separated/widowed & $2,553(40)$ \\
\hline Education, some college, No. (\%) & $3,294(51)$ \\
\hline \multicolumn{2}{|l|}{ Annual income, No. (\%) } \\
\hline$<\$ 20,000$ & $3,769(58)$ \\
\hline$\$ 20,000-\$ 50,000$ & $1,946(30)$ \\
\hline$>\$ 50,000$ & $704(11)$ \\
\hline \multicolumn{2}{|l|}{ 10-item AUDIT score, No. (\%) } \\
\hline 1-7 (zone 1) & $4,083(64)$ \\
\hline 8-15 (zone 2) & $1,543(24)$ \\
\hline 16-19 (zone 3) & $290(5)$ \\
\hline 20-40 (zone 4) & $503(8)$ \\
\hline \multicolumn{2}{|l|}{ AUDIT-C score, No. (\%) } \\
\hline $1-3$ & $2,427(38)$ \\
\hline $4-5$ & $1,749(27)$ \\
\hline $6-7$ & $1,031(16)$ \\
\hline $8-9$ & $667(10)$ \\
\hline $10-12$ & $545(9)$ \\
\hline \multicolumn{2}{|l|}{ CAGE score, No. (\%) } \\
\hline 0 & $1,595(25)$ \\
\hline 1 & $1,424(22)$ \\
\hline 2 & $1,598(25)$ \\
\hline 3 & $1,095(17)$ \\
\hline 4 & 707 (11) \\
\hline \multicolumn{2}{|c|}{$\begin{array}{l}\text { AUDIT = Alcohol Use Disorders Identification Test; AUDIT-C }=3 \text { AUDIT con- } \\
\text { sumption questions; CAGE = CAGE Questionnaire (cut down, annoyed, guilty, } \\
\text { and eye opener). }\end{array}$} \\
\hline \multicolumn{2}{|l|}{$\begin{aligned}{ }^{*} \mathrm{n} & =6,261, \text { missing data for } 158 \text { subjects. } \\
t \mathrm{n} & =6,269, \text { missing data for } 150 \text { subjects. }\end{aligned}$} \\
\hline
\end{tabular}


indicated they no longer drank alcohol ( $\mathrm{n}=389)$, study participants were slightly older (59.5 vs 58.1 years); were more likely to be white (64\% vs $53 \%$ ), married (49\% vs $43 \%)$, retired (39\% vs 35\%), and to have attended college $(51 \%$ vs $46 \%)$; and reported incomes greater than $\$ 20,000$ per year $(41 \%$ vs $36 \%)(P<.001$ for all measures). Participants were also more likely than eligible nonrespondents to the Drinking Practices Questionnaire to have a positive score on the AUDIT-C ( $\geq 4$ points) $(67 \%$ vs $58 \% ; P<.001)$ and less likely to score 2 or more points on the CAGE $\left(53 \%\right.$ vs $\left.60 \%{ }_{i} P<.001\right)$ on the initial Health Checklist questionnaire.

The 6,419 respondents reflected a broad spectrum of alcohol misuse severity based on responses to all 3 measures, with $36 \%$ of patients having AUDIT scores $\geq 8$. According to their responses to the readiness-tochange questions, $25 \%$ were categorized into precontemplation, $24 \%$ into contemplation, and $51 \%$ into action. When readiness groups were cross-tabulated with alcohol misuse severity measures, there were significant, positive linear-by-linear associations between readiness groups and alcohol misuse severity $(P<.001)$ (Table 2).

Because of the algorithm used to define the precontemplation and action groups (Figure 1), analyses of individual responses to readiness-to-change questions among patients categorized into these groups showed relatively homogenous responses (data not presented). Patients who were categorized into the contemplation group, however, had potentially important variation in

responses to the 3 individual readiness-to-change questions. Specifically, the proportion of patients in the contemplation group who reported "I am sure I drink more than I should" increased as the severity of alcohol misuse increased: $4 \%$ in AUDIT zone 1 (AUDIT scores $<8$ ); $16 \%$ in zone 2 (AUDIT scores 8 to 15 ); $39 \%$ in AUDIT zone 3 (AUDIT scores 16 to 19); and $69 \%$ in zone 4 (AUDIT scores $>20$ ). Similarly, higher severity of alcohol misuse was associated with increasing percentages of patients in the contemplation group reporting wanting but not having been able to reduce drinking: $3 \%$ in AUDIT zone $1 ; 10 \%$ in AUDIT zone $2 ; 27 \%$ in AUDIT zone $3 ;$ and $60 \%$ in AUDIT zone 4.

\section{DISCUSSION}

In this population of male VA primary care patients who screened positive for alcohol misuse in the past year, $75 \%$ indicated some readiness to change. Contrary to a stereotype that patients with alcohol misuse will deny concerns about their drinking, most patients whose tests were positive for alcohol misuse in this population indicated they drank more than they should or had considered or tried decreasing drinking. Moreover, readiness to change increased steadily as the severity of alcohol misuse increased. Among patients who scored 8 or more on the AUDIT, indicative of a high likelihood of active alcohol use disorders in this population, ${ }^{34,35}$ readiness to change was relatively stable, with more than $90 \%$ of patients indicating that they drank excessively or

\section{Table 2. Participants Categorized Into Readiness to Change Groups Stratified by Alcohol Screening Scores}

\begin{tabular}{|c|c|c|c|c|c|}
\hline $\begin{array}{l}\text { Alcohol Misuse } \\
\text { Severity Score }\end{array}$ & $\begin{array}{c}\text { Precontemplation } \\
\text { n (\%) }\end{array}$ & $\begin{array}{c}\text { Contemplation } \\
\text { n (\%) }\end{array}$ & $\begin{array}{l}\text { Action } \\
\text { n (\%) }\end{array}$ & Total & $\begin{array}{c}P \\
\text { Value }\end{array}$ \\
\hline AUDIT & & & & & $<.001$ \\
\hline$<8$ & 1,518 (37) & 631 (15) & $1,934(47)$ & 4,083 & \\
\hline $8-15$ & $96(6)$ & 535 (35) & 912 (59) & 1,543 & \\
\hline $16-19$ & $5(2)$ & $122(42)$ & $163(56)$ & 290 & \\
\hline$>20$ & $3(1)$ & $245(49)$ & $255(51)$ & 503 & \\
\hline AUDIT-C & & & & & $<.001$ \\
\hline $1-3$ & 956 (39) & $167(7)$ & $1,304(54)$ & 2,427 & \\
\hline $4-5$ & 497 (28) & $444(25)$ & $808(46)$ & 1,749 & \\
\hline $6-7$ & $113(11)$ & $362(35)$ & $556(54)$ & 1,031 & \\
\hline $8-9$ & $36(5)$ & $278(42)$ & $353(53)$ & 667 & \\
\hline $10-12$ & $20(4)$ & $282(52)$ & $243(45)$ & 545 & \\
\hline CAGE & & & & & $<.001$ \\
\hline 0 & $672(42)$ & 297 (19) & $626(39)$ & 1,595 & \\
\hline 1 & $283(20)$ & $386(27)$ & 755 (53) & 1,424 & \\
\hline 2 & $357(22)$ & $418(26)$ & $823(52)$ & 1,598 & \\
\hline 3 & 197 (18) & $272(25)$ & $626(57)$ & 1,095 & \\
\hline 4 & $113(16)$ & $160(23)$ & $434(61)$ & 707 & \\
\hline
\end{tabular}

had considered change (Figure 2).

Findings of the present study were consistent with those of 2 previous primary care studies. ${ }^{11,17,18}$ Both required that patients provide written informed consent to participate in a study of their drinking. One study was small, and $56 \%$ of the 184 participating patients who had positive CAGE scores no longer drank alcohol. ${ }^{18}$ The other study used a sample of patients recruited for a randomized controlled trial of brief alcohol counseling interventions. ${ }^{11}$ In that study, $78 \%$ of patients who had positive test results did not participate in the 


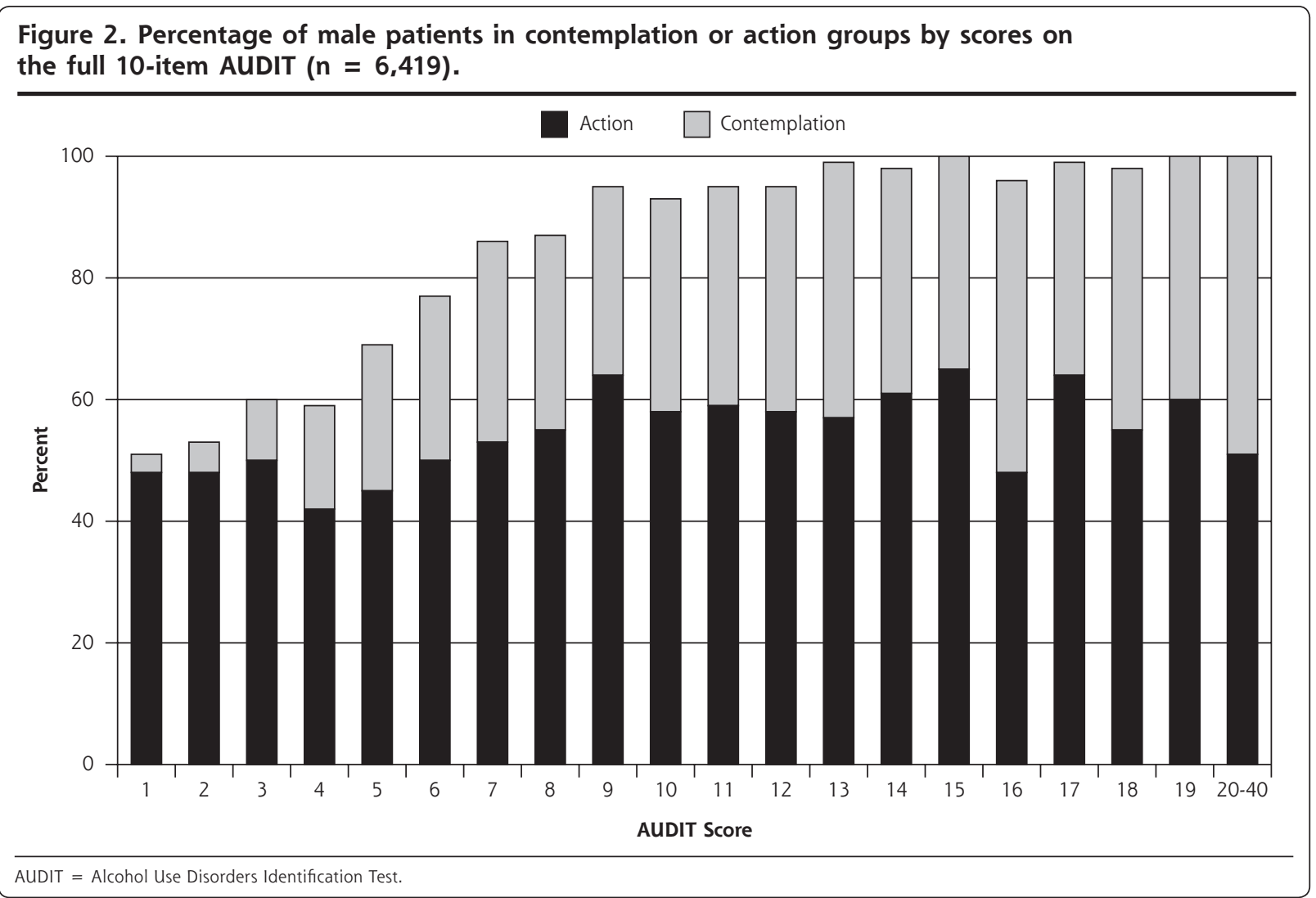

trial, potentially limiting the generalizability of their findings. Our study is the first to describe readiness to change in a large sample of primary care patients who screened positive for alcohol misuse but were not being recruited into a study focusing exclusively on their drinking.

This study has several noteworthy limitations. First, our population consisted of male VA patients who were predominantly white and older, which may limit the generalizability of our findings to women and other primary care populations. Second, nonresponse bias could have influenced our findings. Forty-five percent of patients who screened positive for alcohol misuse on the initial ACQUIP survey were not included in these analyses. Nonparticipants were more likely to screen positive for a lifetime history of alcohol abuse or dependence on the CAGE, but were less likely to report high levels of current consumption on the AUDIT-C. Lower AUDIT-C scores among nonrespondents could reflect underreporting of alcohol use. Even in the unlikely event that all nonrespondents to the Drinking Practices Questionnaire were in the precontemplation group, however, almost one half of the resulting sample would still have been classified in contemplation or action. Social desirability could also have accounted for some of our findings. Patients who were not interested in changing might minimize their alcohol misuse and related problems or overreport recent decreases in their drinking so that their primary care clinicians would not bother them about their drinking. Finally, the algorithm used to measure readiness to change in this study has been validated only in a female VA population. ${ }^{28}$

The study also has several important strengths. First, the size of the sample is a strength, as is that more than $10 \%$ of the entire eligible study sample screened positive for alcohol misuse and returned the Drinking Practices Questionnaire. Additionally, the prevalences of self-reported alcohol misuse and related problems were high, decreasing the likelihood that high rates of readiness to change reflected social desirability bias and providing rich variation in alcohol misuse among participants. Finally, although the readiness-to-change questions have been validated in only women veterans, the brevity and clinical accessibility of this instrument makes it one of our study's unique strengths.

This study indicates that most primary care patients who screened positive for alcohol misuse and who returned a questionnaire that assesses alcohol misuse had some recognition that they drink more than they should and/or have at least contemplated drinking less. Moreover, as screening scores increased, 
patients were more likely to report wanting but having been unable to decrease drinking. These findings have several implications for clinicians. First, primary care clinicians sometimes expect patients to deny their alcohol misuse when the issue is raised. ${ }^{13-16}$ Such denial is often thought to be especially common among patients with the most-severe problems, indicative of alcohol dependence. ${ }^{14}$

Our findings support findings of previous studies in more-select populations that suggest the opposite is true. The more a patient drinks and the more severe his problems caused by drinking, the more likely he will report recognition of or interest in changing his drinking. A recent study of clinicians' attitudes and their association with smoking cessation counseling reported that clinicians' perceptions that patients are not willing to quit smoking were associated with a lower counseling proclivity. ${ }^{40}$ Similar clinicians' attitudes may be a barrier to conducting brief alcohol counseling interventions among patients with alcohol misuse. Our finding that most primary care patients with alcohol misuse report some level of readiness to change could help correct such attitudes. Second, these findings suggest that primary care clinicians could use scores obtained from brief alcohol-screening questionnaires as an indicator of readiness to change. Patients whose screening tests are positive but who have low scores are least likely to recognize they drink more than they should or to con-

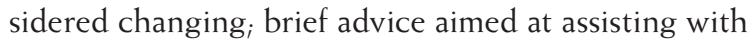
problem recognition and building motivation may be most appropriate for these patients.

Maisto et al found that low levels of readiness to change at baseline were associated with improved drinking outcomes at follow-up for patients who received brief advice. ${ }^{11}$ Taken with our findings, we hypothesize that patients with the least severe alcohol misuse, who are least likely to recognize that they drink excessively or to have contemplated change, may benefit most from brief alcohol-related advice in primary care settings. ${ }^{11}$ This stance is consistent with current evidence-based guidelines that recommend screening for the entire spectrum of alcohol misuse in primary care settings. ${ }^{4,41}$ Appropriate and brief screening tests for this purpose include the AUDIT-C or single-item questions about binge drinking. ${ }^{34,42,43}$

That patients in this study with higher alcoholscreening scores were more likely to indicate recognition of their alcohol misuse or interest in changing should not be confused with readiness to enter specialized alcohol treatment. Many patients in this study's contemplation group reported wanting but having been unable to decrease drinking, which was positively associated with the severity of alcohol misuse. Patients with more-severe alcohol misuse may need more- intense or repeated primary care interventions to support them toward specialized addictions treatment or abstinence. ${ }^{44,45}$ Similarly, it is unknown whether indication of readiness to change by primary care patients will result in subsequent changes in drinking, though patient readiness to change has been associated with decreased drinking in hospitalized patients. ${ }^{27}$ Further research is needed in both of these areas.

In conclusion, this study shows that most primary care patients who screen positive for alcohol misuse indicate concern about or are considering changing their drinking. Further, simple questions can elicit statements reflecting readiness to change, and patients with the highest alcohol-screening scores and greater alcohol misuse severity are most likely to indicate some readiness to change drinking. These findings should help counter clinician attitudes that patients with alcohol misuse deny excessive drinking or will not be interested in changing.

To read or post commentaries in response to this article, see it online at http://www.annfammed.org/cgi/content/full/4/3/213.

Key words: Alcohol drinking; alcoholism/diagnosis; patient acceptance of health care

Submitted June 29, 2005; submitted, revised, October 28, 2005; accepted November 28, 2005.

A preliminary version of this study was presented at the Society of General Internal Medicine annual meeting, Chicago, III, May 14, 2004.

Funding support: The Ambulatory Care Quality Improvement Project (ACQUIP) was funded by Veterans Affairs Health Services Research and Development grants: SDR 96-002 and IIR 99-376. Dr Bradley is supported by a National Institute on Alcohol Abuse and Alcoholism award (K23AA00313) and was a Robert Wood Johnson Generalist Physician Faculty Scholar at the time this work was completed.

Disclaimer: Views expressed in this article are those of the authors and do not necessarily represent the views of the Department of Veterans Affairs, the University of Washington, the National Institute on Alcohol Abuse and Alcoholism, or the Robert Wood Johnson Foundation.

\section{References}

1. Grant BF, Dawson DA, Stinson FS, et al. The 12-month prevalence and trends in DSM-IV alcohol abuse and dependence: United States, 1991-1992 and 2001-2002. Drug Alcohol Depend. 2004;74:223-234.

2. Tenth Special Report to the US Congress on Alcohol and Health. US Department of Health and Human Services. National Institute on Alcohol Abuse and Alcoholism; 2000.

3. Whitlock EP, Polen MR, Green CA, Orleans T, Klein J. Behavioral counseling interventions in primary care to reduce risky/harmful alcohol use by adults: a summary of the evidence for the U.S. Preventive Services Task Force. Ann Intern Med. 2004;140:557-568.

4. Screening and behavioral counseling interventions in primary care to reduce alcohol misuse: recommendation statement. Ann Intern Med. 2004;140:554-556.

5. Saitz R. Clinical practice. Unhealthy alcohol use. N Engl J Med. 2005;352:596-607. 
6. Babor TF, Higgins-Biddle JC. Alcohol screening and brief intervention: dissemination strategies for medical practice and public health. Addiction. 2000;95:677-686.

7. US Department of Health and Human Services. National Institute on Alcohol Abuse and Alcoholism. Helping Patients With Alcohol Problems: A Practitioner's Guide. Bethesda, Md: National Institute of Health; 2003.

8. Rustin TA. Assessing nicotine dependence. Am Fam Physician. 2000;62:579-584, 591-572.

9. Dijkstra A, De Vries H, Roijackers J. Targeting smokers with low readiness to change with tailored and nontailored self-help materials. Prev Med. 1999;28:203-211.

10. Samet JH, Rollnick S, Barnes H. Beyond CAGE. A brief clinical approach after detection of substance abuse. Arch Intern Med. $1996 ; 156: 2287-2293$.

11. Maisto SA, Conigliaro J, McNeil M, et al. Effects of two types of brief intervention and readiness to change on alcohol use in hazardous drinkers. J Stud Alcohol. 2001;62:605-614.

12. Heather N, Rollnick S, Bell A, Richmond R. Effects of brief counseling among male heavy drinkers identified on general hospital wards. Drug Alcohol Rev. 1996;15:29-38.

13. Beich A, Gannik D, Malterud K. Screening and brief intervention for excessive alcohol use: qualitative interview study of the experiences of general practitioners. BMJ. 2002;325:870.

14. Arborelius E, Damstrom Thakker K. Why is it so difficult for general practitioners to discuss alcohol with patients? Fam Pract. $1995 ; 12: 419-422$.

15. Thom B, Tellez C. A difficult business: detecting and managing alcohol problems in general practice. Br J Addict. 1986;81:405-418

16. Aira M, Kauhanen J, Larivaara P, Rautio P. Factors influencing inquiry about patients' alcohol consumption by primary health care physicians: qualitative semi-structured interview study. Fam Pract. 2003:20:270-275.

17. Maisto SA, Conigliaro J, McNeil M, et al. Factor structure of the SOCRATES in a sample of primary care patients. Addict Behav. 1999;24:879-892.

18. Samet JH, O'Connor PG. Alcohol abusers in primary care: readiness to change behavior. Am J Med. 1998;105:302-306.

19. Fihn SD, McDonell MB, Diehr P, et al. Effects of sustained audit/ feedback on self-reported health status of primary care patients. Am J Med. 2004;116:241-248.

20. Bradley KA, Kivlahan DR, Bush KR, et al. Variations on the CAGE alcohol screening questionnaire: strengths and limitations in VA general medical patients. Alcohol Clin Exp Res. 2001;25:1472-1478.

21. Bradley KA, Boyd-Wickizer J, Powell S, Burman ML. Alcohol screening questionnaires in women: a critical review. JAMA 1998:280:166-171

22. DiClemente CC, Schlundt D, Gemmell L. Readiness and stages of change in addiction treatment. Am J Addict. 2004;13:103-119.

23. Prochaska JO, DiClemente CC. Transtheoretical therapy: toward a more integrative model of change. Psychother Theory Res Prac. 1982; 19:276-288.

24. Vik PW, Culbertson KA, Sellers K. Readiness to change drinking among heavy-drinking college students. J Stud Alcohol. $2000 ; 61: 674-680$

25. Isenhart CE. Pretreatment readiness for change in male alcohol dependent subjects: predictors of one-year follow-up status. I Stud Alcohol. 1997;58:351-357.

26. Dent TH, Shepherd RM, Alexander GJ, London M. Do CAGE scores predict readiness to reduce alcohol consumption in medical inpatients? Alcohol Alcohol. 1995;30:577-580.
27. Heather N, Rollnick S, Bell A. Predictive validity of the Readiness to Change Questionnaire. Addiction. 1993;88:1667-1677.

28. Epler AJ, Kivlahan DR, Bush KR, Dobie DJ, Bradley KA. A brief readiness to change drinking algorithm: concurrent validity in female VA primary care patients. Addict Behav. 2005;30:389-395.

29. Prochaska JO, DiClemente CC. Stages and processes of self-change of smoking: toward an integrative model of change. J Consult Clin Psychol. 1983;51:390-395.

30. Rollnick S, Heather N, Gold R, Hall W. Development of a short 'readiness to change' questionnaire for use in brief, opportunistic interventions among excessive drinkers. Br J Addict. 1992;87:743-754

31. Saunders JB, Aasland OG, Babor TF, de la Fuente JR, Grant M. Development of the Alcohol Use Disorders Identification Test (AUDIT): WHO Collaborative Project on Early Detection of Persons with Harmful Alcohol Consumption--II. Addiction. 1993;88:791-804.

32. Bradley KA, Bush KR, McDonell MB, Malone T, Fihn SD. Screening for problem drinking: comparison of CAGE and AUDIT. Ambulatory Care Quality Improvement Project (ACQUIP). Alcohol Use Disorders Identification Test. J Gen Intern Med. 1998;13:379-388.

33. Babor TF, Higgins-Biddle JC, Saunders JB, Monteiro M. AUDIT - The Alcohol Use Disorders Identification Test: Guidelines for Use in Primary Health Care. 2nd ed. World Health Organization, Department of Mental Health and Substance Abuse; 2001. Report No.: WHO/ MSD/MSB/01.6a

34. Bush K, Kivlahan DR, McDonell MB, Fihn SD, Bradley KA. The AUDIT alcohol consumption questions (AUDIT-C): an effective brief screening test for problem drinking. Ambulatory Care Quality Improvement Project (ACQUIP). Alcohol Use Disorders Identification Test. Arch Intern Med. 1998;158:1789-1795.

35. Bradley KA, McDonell MB, Bush K, et al. The AUDIT alcohol consumption questions: reliability, validity, and responsiveness to change in older male primary care patients. Alcohol Clin Exp Res. $1998 ; 22: 1842-1849$

36. Bradley KA, Kivlahan DR, Zhou XH, et al. Using alcohol screening results and treatment history to assess the severity of at-risk drinking in Veterans Affairs primary care patients. Alcohol Clin Exp Res. 2004:28:448-455

37. Adams WL, Barry KL, Fleming MF. Screening for problem drinking in older primary care patients. JAMA. 1996;276:1964-1967.

38. Fiellin DA, Reid MC, O'Connor PG. Screening for alcohol problems in primary care: a systematic review. Arch Intern Med. 2000;160:1977-1989.

39. SPSS for Windows [computer program]. Version 10.0.7. Chicago, Ill: SPSS Inc; 1999.

40. Meredith LS, Yano EM, Hickey SC, Sherman SE. Primary care provider attitudes are associated with smoking cessation counseling and referral. Med Care. 2005;43:929-934.

41. US Department of Health and Human Services. National Institute on Alcohol Abuse and Alcoholism. Helping Patients Who Drink Too Much: A Clinician's Guide. Bethesda, Md: National Institute of Health; 2005.

42. Taj N, Devera-Sales A, Vinson DC. Screening for problem drinking: does a single question work? J Fam Pract. 1998;46:328-335.

43. Williams R, Vinson DC. Validation of a single screening question for problem drinking. J Fam Pract. 2001;50:307-312.

44. Lieber CS, Weiss DG, Groszmann R, Paronetto F, Schenker S. I. Veterans Affairs Cooperative Study of polyenylphosphatidylcholine in alcoholic liver disease: effects on drinking behavior by nurse/physician teams. Alcohol Clin Exp Res. 2003;27:1757-1764.

45. Willenbring $\mathrm{ML}$, Olson $\mathrm{DH}$. A randomized trial of integrated outpatient treatment for medically ill alcoholic men. Arch Intern Med. 1999;159:1946-1952. 